\title{
Thermo-electric model of a pyroelectric detector
}

\author{
K. Domke \& A. Odon \\ Poznań University of Technology, Institute of Electrical Engineering and \\ Electronics, Poland
}

\begin{abstract}
This paper discusses the construction and operating rules of a pyroelectric detector. An analysis of heat phenomena occurring in the detector and the influence on the signals generated by the detector was carried out. A strict dependence of the electric characteristics of the detector upon its heat properties determined by the geometrical structure and heat properties of the materials that make up the detector was emphasised, while considering the detectors used for the conversion of impulse signals. Also emphasised was the necessity to take into account the differences occurring in the process of heating up and cooling down of the pyroelectric film of the detector.
\end{abstract}

Keywords: pyroelectric detector, thermal model, heat exchange in detector.

\section{Introduction}

Pyroelectric detectors play an important role in such applications as infrared radiation detection, measurements of emission parameters of radiation sources, remote measurements of temperature as well as heat and visual measurements. Information concerning the measurable radiation values is included in the parameters of the pyroelectric detector current or voltage response to heat agitation by the energy provided by the radiation absorbed by the detector. Multiple applications of this detector stimulate interest of a number of research teams in both new pyroelectric detectors manufacturing and improved analytic description, especially the one related to the pyroelectric detector modelling.

A pyroelectric detector is a heat converter that enables transformation of the absorbed radiation into an increase of temperature of the pyroelectric material and subsequently into a measurable electric signal. During the process of 
pyroelectric detector modelling, it is therefore important to take into account those parameters of a heat circuit that have a material influence on heat effects. Improper selection or too many factors and parameters of the heat transfer process taken into account may result in a not very reliable or a highly complex model of a pyroelectric detector, which in turn may render simulation research difficult or even impossible. It is worth observing that generally topics related to the analysis of heat effects in the operation of the pyroelectric detectors are rather poorly represented in the available literature.

\section{Structure and operation of the pyroelectric detector}

Figure 1 represents the structure of a pyroelectric detector.

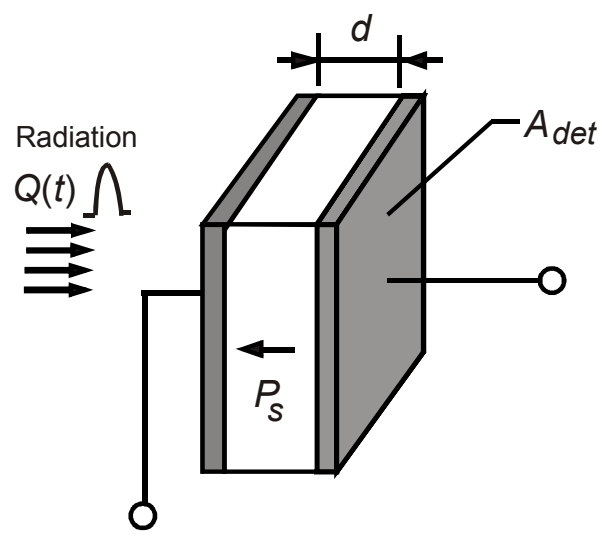

Figure 1: Structure of the pyroelectric detector.

In principle, from the point of view of its construction, the pyroelectric detector is a capacitor with capacitance $C_{d}$. The capacitor's dielectric is made from a thin plate of a pyroelectric material having a thickness $d$ and surface $A_{\operatorname{det}}$ coated with a metallised film. If the varying in time radiation of the power $Q(t)$ is incident on the active surface of the pyroelectric detector, then as a result of the heat and electric processes occurring in the pyroelectric material having a spontaneous polarisation $P_{S}$ an electric charge $q(t)$ will appear. The signals bearing the information about radiation signal parameters may be both the voltage $V(t)$ on the pyroelectric detector and the $I_{p}(t)$ current flowing through the pyroelectric detector load circuit with very low impedance. Three stages can be identified in the process of conversion of the absorbed radiation into an electric signal, i.e. radiation and heat conversion of the radiation with the momentary power $Q(t)$ incident on the detector surface into the change of the temperature of the pyroelectric material of the detector $\Delta T(t)$, heat and electric conversion consisting in the conversion of temperature changes $\Delta T(t)$ in the pyroelectric into the electric current of a current source $I_{p}(t)$ and electric conversion of a current 
signal $I_{p}(t)$ into a voltage signal $V(t)$ arriving at the voltage amplifier input. Fig. 2 illustrates the discussed signal conversion stages and the corresponding equivalent schematic drawing of the pyroelectric detector cooperating with the voltage amplifier.
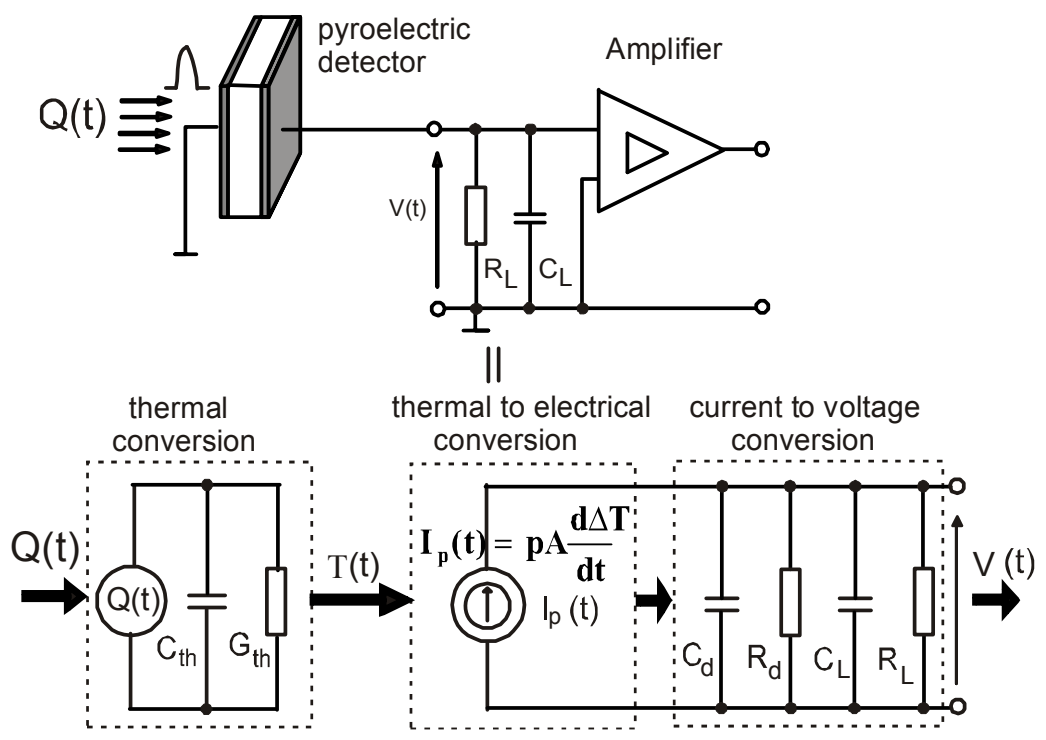

Figure 2: Equivalent scheme of a pyroelectric detector.

where:

$C_{t h}-$ thermal capacity of pyroelectric detector,

$G_{t h}$ - thermal conductance of pyroelectric detector,

$p$ - pyroelectric coefficient,

$C_{d}$ and $C_{L}$ - electrical capacity of pyroelectric detector and capacity of amplifier input respectively

$R_{d}$ and $R_{L}$ - leakage resistance of pyroelectric detector and input amplifier resistance respectively

\section{Thermal models of the pyroelectric detector}

In the related literature (Efthymiou et al. $[1,2]$ ), it is assumed that the heat and electric properties of the detector depend on the heat characteristics of the detector head. Thus, only this part of the entire detector is considered during the field modelling or construction of the equivalent heat schematic drawings.

Usually, the heat models of the pyroelectric detector are presented as analogue heat drawings with solid elements or $3 \mathrm{D}$ objects including a detection component, support legs and films. Figure 3 illustrates a simplified view of a typical structure of the pyroelectric detector. 


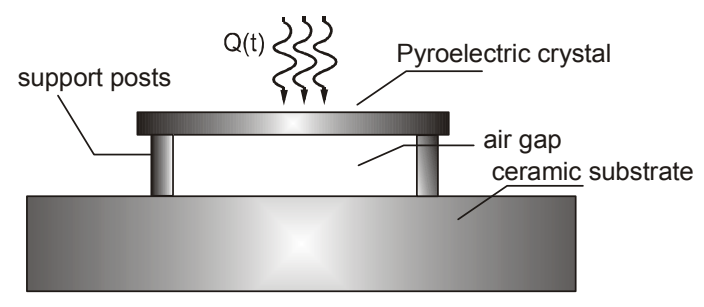

Figure 3: $\quad$ Structure of a pyroelectric detector.

An equation describing a one-dimensional heat transfer is usually represented as:

$$
C_{t h} \frac{d \Delta T(t)}{d t}+\frac{\Delta T(t)}{R_{t h}}=a \cdot Q(t)
$$

where $R_{t h}$ and $C_{t h}$ are the heat resistance and the thermal capacity respectively, $a$ is the absorption coefficient of radiation, $Q$ radiation power stream incident on the detector surface and $a \cdot Q(t)$ - is the flux of absorbed power radiation.

Thermal capacity $C_{t h}$ and heat resistance $R_{t h}$ as given in eqn (1) above are the sum of the elements considered in the heat circuit of the detector in accordance with the dependencies defined in eqns (2-3).

$$
\begin{aligned}
& R_{t h}=\sum_{i} R_{t h-i}=R_{t h-\mathrm{det}}+R_{t h-\text { gap } \| \text { post }}+R_{t h-s u b} \\
& C_{t h}=\sum_{i} C_{t h-i}=C_{t h-\mathrm{det}}+C_{\text {th-gap } \| \text { post }}+C_{t h-s u b}
\end{aligned}
$$

The number of these elements varies depending on the accuracy of the heat model, i.e. identification of the limits of the model considered by the author of a specific paper. It seems, however, that the excessive multiplication of the number of elements taken into account does not increase the accuracy and may even reduce it.

The individual components of eqns (2) and (3) are described with the following dependencies:

$$
\begin{gathered}
C_{t h-i}=V_{i} c^{\prime}{ }_{i}=A_{i} d_{i} \rho_{i} c_{w-i} \\
R_{t h-i}=\frac{d_{i}}{A_{i} \lambda_{i}}
\end{gathered}
$$

where: $A_{i}$ is a crosswise surface, $d_{i}$ thickness, $\rho_{i}$ denotes density and $\lambda_{i}$ is thermal conductivity of layer material of this heat model element.

In the second stage of conversion, due to variations in the temperature of the pyroelectric material, an electric charge appears on the detector electrodes which cause the current to flow in the circuit attached to the detector. It is assumed that the pyroelectric detector can be regarded as temperature $\Delta T(t)$ variation 
controlled current source $I_{p}$, whose value is described with the following dependence:

$$
I_{p}=p A_{\operatorname{det}} \frac{d \Delta T(t)}{d t}
$$

Eqn (6) depicts the fact that an increase of temperature of the pyroelectric material by $\Delta T$ results in the induction of a pyroelectric current $I_{p}$ as described by the eqn (6), whose value is proportional to the velocity of temperature changes of this element in time.

Eqn (1) is usually given as the basic thermal balance equation and in combination with eqn (6) it creates a system used for the modelling of a pyroelectric detector. The corresponding circuit of connections of resistances and thermal capacities is given in fig. 4 .

$$
\Delta T=\sum_{i} \Delta T_{i}
$$

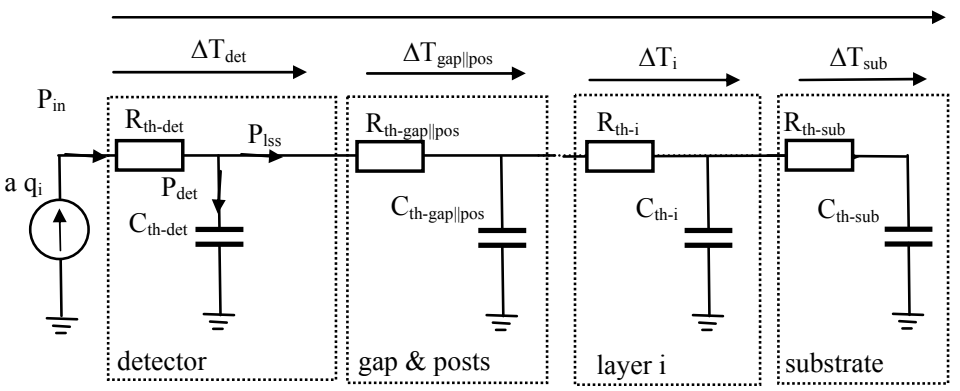

Figure 4: Capacity and heat resistance connections system in the pyroelectric detector.

This system, when using eqns (2)-(3), can be simplified to a simple equivalent schematic drawing as presented in fig. 5 where the thermal resistance was replaced with the heat conductivity $G_{t h}=1 / R_{t h}$.

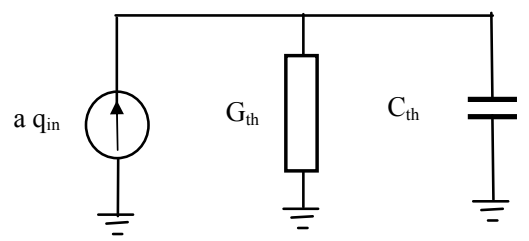

Figure 5: Equivalent schematic drawing of the heat system of the detector head.

In reality, the thermal system and the detector heat stream flows drawing is far more complex as the detector's head elements usually have various types of 


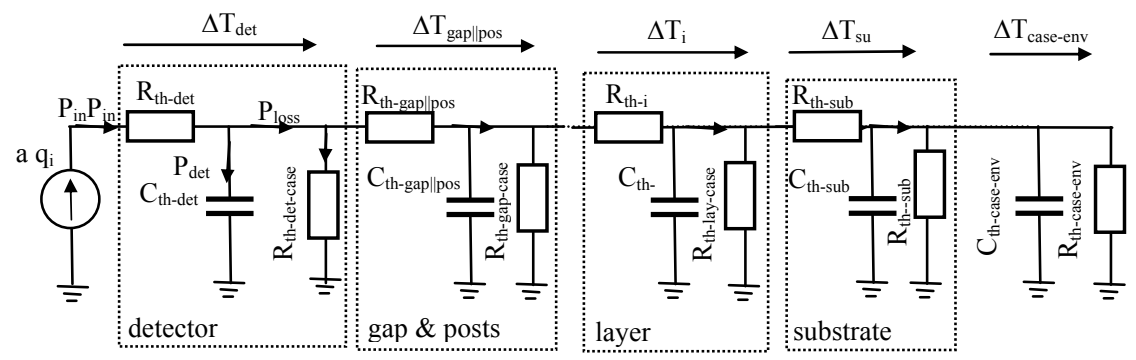

Figure 6: Heat connections schematic drawing taking into account the heat power flow through the detector and between the detector components and encapsulation.

thermal connections to the housing (body) of the entire detector. The connections may be of conductance, convection or radiation type. The heat propagation schematic drawing thus assumes the system configuration as presented in fig. 6 . where heat resistances $R_{\text {th-i-cas }}$ denote the resulting (summary) resistances between these elements of the construction and the housing. Arrows in fig. 6 denote the flow of heat powers essential to the operation of the detector. These powers are grouped on the detection film of the pyroelectric detector in its direct surrounding. It is a consequence of the fact that polarisation and the subsequent occurrence of the current $I_{p}$ concern only the sensitive film of the pyroelectric detector and temperature variations occurring only in this layer. Thus, the correct notation of eqns (1) and (6) should be as follows:

$$
\begin{gathered}
C_{t h-\mathrm{det}} \frac{d \Delta T_{\mathrm{det}}(t)}{d t}+\frac{\Delta T_{\mathrm{det}}(t)}{R_{t h-\operatorname{det}}}=a \cdot Q(t) \\
I_{p}=p A_{\mathrm{det}} \frac{d \Delta T_{\mathrm{det}}(t)}{d t}
\end{gathered}
$$

Other resistances and heat capacities constitute only the external heat load of the pyroelectric film and may be reduced to the following equivalent values $R_{t h-t o t}$ and $C_{\text {th-tot }}$. The resulting heat circuit has therefore the following form shown in fig. 7.

External radiant flux (radiant power) $Q_{\text {in }}$ is incident on the absorption film and absorbed $\left(Q_{a}\right)$; thus, the conversion of the radiant flux into heat energy takes place. It is manifested by the temperature increase of the absorption film. The heat resistance and capacity of the absorption film are very low and may be neglected (Odon [4]). While penetrating the detection layer, the $Q_{a}$ power loads the thermal capacity $C_{t h-d e t}$, and thus contributes to the increase of its temperature $\Delta T_{\text {det }}$.

In order to determine the nature of heating or cooling down of a body, a notion of a thermally thin or thick body has been introduced in thermokinetics [3]. In the first case, the heat resistance of the body is so small that the 


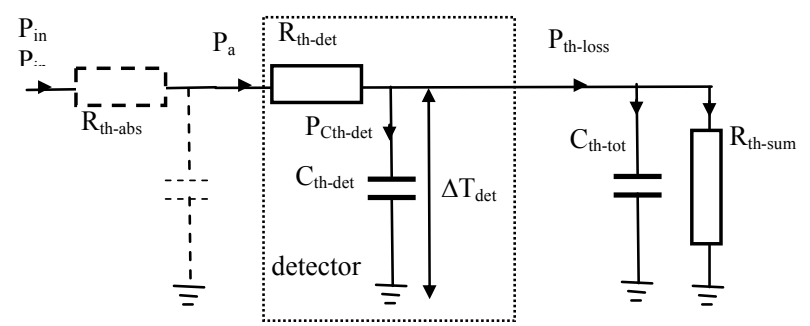

Figure 7: Equivalent schematic drawing of the detector head heat circuit. Heat flow directions in the pyroelectric detector head corresponding to this drawing in the real head are illustrated in fig. 8 .

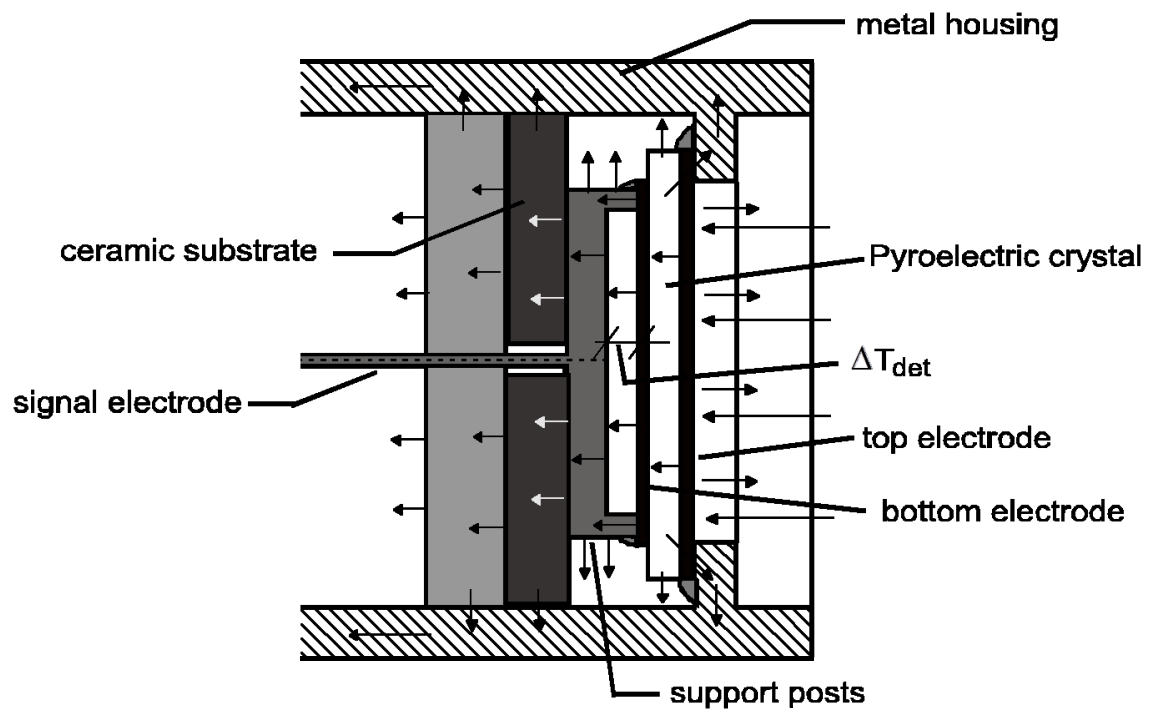

Figure 8: Heat power flow in the pyroelectric head.

temperature distribution field inside this body is - with the exactitude up to $3 \%-$ homogeneous. In other words, each point in the body has the same temperature, which considerably simplifies the calculation and does not require determination of temperature distribution field inside the body. A criterion allowing us to identify whether the body in question falls into the thermally thin category is the value of the Biot number $\boldsymbol{B i}$, defined as internal heat resistance to heat resistance of the external surface heat absorption ratio (9).

$$
B i=\frac{R_{\text {th-in }}}{R_{\text {th-out }}}=\frac{\frac{d}{\lambda \cdot A}}{\frac{1}{\alpha_{\text {rad }} \cdot A}}=\frac{d}{\lambda \cdot \alpha_{\text {rad }}}
$$


For $B i<0,1$, it is assumed that the bodies may be treated as thermally thin bodies (Modest [5]).

For a typical presentation in literature, $R_{\text {th-out }}$ is the heat resistance of the convection heat absorption [5], while with regard to the discussed cases of pyroelectric detectors $R_{\text {th-out }}$ should be interpreted as the resistance of the traditional heat absorption on the absorption surface of the detector. In this case, the radiation heat absorption ratio $\alpha_{\text {rad }}$ can be depicted by the following formula

$$
\alpha_{\text {rad }}=\sigma \cdot A \cdot \frac{T_{\mathrm{det}}^{4}-T_{a m b}^{4}}{T_{\mathrm{det}}-T_{a m b}}
$$

where $\sigma=5,66910^{-8} \mathrm{~W} / \mathrm{m}^{2} \mathrm{~K}^{4}$ is the Stefan-Boltzmann constant.

The analysis of formula (10) emphasises the fact that the $\alpha_{\text {rad }}$ value increases as the $T_{d e t}-T_{a m b}$ temperature difference increases. The exact calculation of these values is practically impossible (lack of data); however, the $T_{\text {Curie }}$ value can be used as an a priori calculation of the $T_{\text {det }}$ value.

Taking the ambient temperature value $T_{a m b}=20^{\circ} \mathrm{C}$, the model values for the presented in (Li et al. [3]) pyroelectric detector head will be as follows $\alpha_{\text {rad }} \approx 6.8$ $\mathrm{W} / \mathrm{m}^{2} \mathrm{~K}$ and $\mathrm{Bi} \approx 0.12 \cdot 10^{-2}$. As other pyroelectric detectors have a similar construction and the condition determined as $B i<0.1$ is satisfied with a considerable margin, for the purpose of further consideration it has been taken that the pyroelectric film of the detector including the sputtered absorption films and conducting electrodes should be treated as a thermally thin body.

In this case, the relative temperature increase $\theta_{\operatorname{det}}(t)$ of the detector as the time function in response to the leap change in the radiation flux is described by the following formula:

$$
\theta_{\mathrm{det}}(t)=\theta_{\mathrm{det}} e^{-\frac{t}{T_{t h}}}
$$

where: $\theta(t)$ denotes the relative temperature difference described by the following dependence:

$$
\theta_{\text {det }}(t)=\frac{T(t)-T(\infty)}{T(0)-T(\infty)}
$$

Thermal time constant $T_{t h}$ of the circuit is determined by the following formula:

$$
T_{t h}=R_{t h} \cdot C_{t h}=\frac{\rho_{w} \cdot c_{w} \cdot d^{2}}{\lambda}
$$

The analysis of fig. 6-8 clearly shows that while considering the detector response to the impulse force, a separation should be made between the warming 
up process of the pyroelectric film of the detector (i.e. the charging of the capacitor $C_{t h \text {-det }}$ through heat resistance $\left.R_{t h-d e t}\right)$ with and the power $P_{C t h \text {-det }}$, from the cooling down process of the pyroelectric film of the detector (i.e. discharge of the capacitor $C_{t h-d e t}$ through heat resistance $R_{\text {th-case }}$ and other resistances presented as summary values $R_{t h-s u m}$ and $C_{t h \text {-sum }}$ in fig. 7 with the power $P_{t h-l o s s}$ ). Resistances and heat capacities are responsible for cooling down of the pyroelectric film and are usually considerably higher than the active warming up resistances and capacities.

Thus, a typical pyroelectric detector is characterised with two different time constants applicable to the pyroelectric film warming up process $T_{t h-H}$ responsible for the growing response of the detector to the impulse force and the time constant of the cooling down process $T_{t h-C}$ responsible for the fading of the detector output voltage.

\section{Summary}

1. The electric characteristics of the pyroelectric detectors are strictly dependent on the construction details (geometry) of these detectors and more particularly on the location of the detection film in relation to other elements of the detector head.

2. The characteristics of the pyroelectric detectors are materially influenced by the propagation of heat fluxes in the direct neighbourhood of the detection film.

3. With respect to detectors for measuring repeatable radiant fluxes, attempts should be made to achieve constant time values of warming up and cooling down of the pyroelectric film.

\section{References}

[1] Efthymiou S, Ozanyan K.Efthymiou S, Ozanyan K. B.: Pulsed performance of pyrpelectric detectors. Journal of Physics. ser. Conferences 178, (2009) 012044.

[2] Efthymiou S, Ozanyan K.Efthymiou S, Ozanyan K. B.: Sensing of Pulsed Radiation with Pyroelectric Detectors. IEEE Sensors 2010, Conference.

[3] Li Bo, Wu Xiaoqing, Yao Xi, Modelling multilayer pyroelectric thin film infrared detector using PSPICE, Integrated Ferroelectrics, 44, pp. 9-18, 2010.

[4] Odon A.: Badanie i przetwarzanie odpowiedzi detektora piroelektrycznego na potrzeby pomiarów energii promienistej (pol.) Wyd. Polit. Poznańskiej, Poznań, 2010.

[5] Modest M. F. Radiative heat transfer, ed. II, Academic Press, N. York, Oxford, Tokyo, 2003 